\title{
Influence of Lymphocytes in Malignant Pleural Effusion on the Therapeutic Efficacy of Intrapleural OK-432 in Lung Cancer Patients
}

\author{
Tetsuo Shimizu, Noriaki Takahashi, Masahiro Terakado, Hiroshi Akusawa, \\ Ichiro Tsujino and Takashi Horie
}

\begin{abstract}
Objectives Malignant pleural effusion, a common complication seen in advanced lung cancer patients, is often treated with intrapleural administration of chemical agents. In Japan, OK-432, a biological response modifiers, which activates the cytotoxic activity of lymphocytes and boosts antitumor immunity, is among the most frequently used chemical agents. The purpose of this study was to determine, in a case-control study, whether or not the rate of lymphocytes in malignant pleural effusion (lymphocyte rate) influences the therapeutic efficacy of intrapleural OK-432.

Patients and Methods We enrolled 20 lung cancer patients with malignant pleural effusion treated with intrapleural OK-432 who were admitted to our hospital between January 2000 and December 2004. Therapeutic efficacy was assessed from the response rate, duration of chest drainage after treatment with intrapleural OK-432, time to progression of malignant pleural effusion, and survival time.

Results Response rate in patients who had a high lymphocyte rate (the High lymphocyte rate group) was significantly higher than in patients who had a low lymphocyte rate (the Low lymphocyte rate group). Lymphocyte rate did not correlate with duration of chest drainage after treatment with intrapleural OK-432, time to progression of malignant pleural effusion, or survival time.

Conclusions The lymphocyte rate in malignant pleural effusion influences the response rate to treatment by intrapleural OK-432. In the High lymphocyte rate group, intrapleural OK-432 for malignant pleural effusion was effective. We conclude that intrapleural OK-432 is useful for malignant pleural effusion patients with a high lymphocyte rate before treatment.
\end{abstract}

Key words: lung cancer, lymphocyte, malignant pleural effusion, OK-432, response rate, tumor-infiltrating lymphocytes

(DOI: 10.2169/internalmedicine.45.1538)

\section{Introduction}

Malignant pleural effusion is a condition in which cancer cells infiltrate or metastasize at the pleura, causing an inflammatory reaction at the pleura, and as a result of which exudative pleural effusion collects in the thoracic cavity. The cell components in malignant pleural effusion contain immunocytes such as lymphocytes and histiocytes in addition to cancer cells. Generally, lymphocytes in the tumor and stroma which show an antitumor immune response are called tumor-infiltrating lymphocytes (TILs) (1). Lymphocytes and cancer cells are mixed in malignant pleural effusion. We suspect that lymphocytes which react to cancer cells in the same way as TILs are related to antitumor immunity.

In primary lung cancer, malignant pleural effusion is typical of the complications seen in advanced lung cancer patients, most of whom complain of dyspnea. Although the therapy for advanced lung cancer is mainly comprised of 
Table 1. Patient Characteristics

\begin{tabular}{|c|c|}
\hline Characteristics & Patients \\
\hline $\mathrm{N}$ & 20 \\
\hline Age yrs (mean) & 66.1 \\
\hline (range) & $(48 \sim 83)$ \\
\hline Gender Male & 12 \\
\hline Female & 8 \\
\hline Stage & 8 \\
\hline $\mathrm{IV}$ & 12 \\
\hline \multicolumn{2}{|c|}{ Histological classification } \\
\hline $\mathrm{Ad}$ & 17 \\
\hline Others & 3 \\
\hline PS $\leqq 2$ & 14 \\
\hline$\geqq 3$ & 6 \\
\hline \multicolumn{2}{|l|}{ Posterior treatment } \\
\hline $\mathrm{BSC}$ & 9 \\
\hline Chemotherapy & 11 \\
\hline
\end{tabular}

Ad: adenocarcinoma

PS: performance status of the Eastern Cooperative Oncology Group BSC: best supportive care

systemic chemotherapy, the effects of systemic chemotherapy on malignant pleural effusion in non-small cell lung cancer, which is the commonest type of primary lung cancer, are low. Malignant pleural effusion in non-small cell lung cancer is often treated by intrapleural administration of a chemical agent to decrease a pleural effusion (2). In Japan, OK-432, a type of biological response modifiers, is among the most frequently used chemical agents (3). It is a streptococcal preparation treated with penicillin. It activates lymphocytes (4), macrophages (4), neutrophils (5), natural killer cells (6), and cytotoxic cells (7) and the lymphocytes produce cytokines such as IL-2 (8), IL-8 (9), IL-12 (10), and IFN- $\gamma(11)$. It energizes the antitumor immunity, giving an antitumor effect. Anticancer drugs such as cisplatin (12) are used for intrapleural administration as well as OK-432, but cisplatin is not much different from OK-432 in therapeutic efficacy. OK-432 is more commonly used, since its toxicity is lower than cisplatin. When OK-432 is administrated to the thoracic cavity, it activates lymphocytes in malignant pleural effusion and has an antitumor effect. To date, the relationship between lymphocytes in malignant pleural effusion and the therapeutic efficacy of intrapleural OK-432 has not been known. The purpose of this study was to determine whether or not lymphocytes in malignant pleural effusion influence the therapeutic efficacy of intrapleural OK-432.

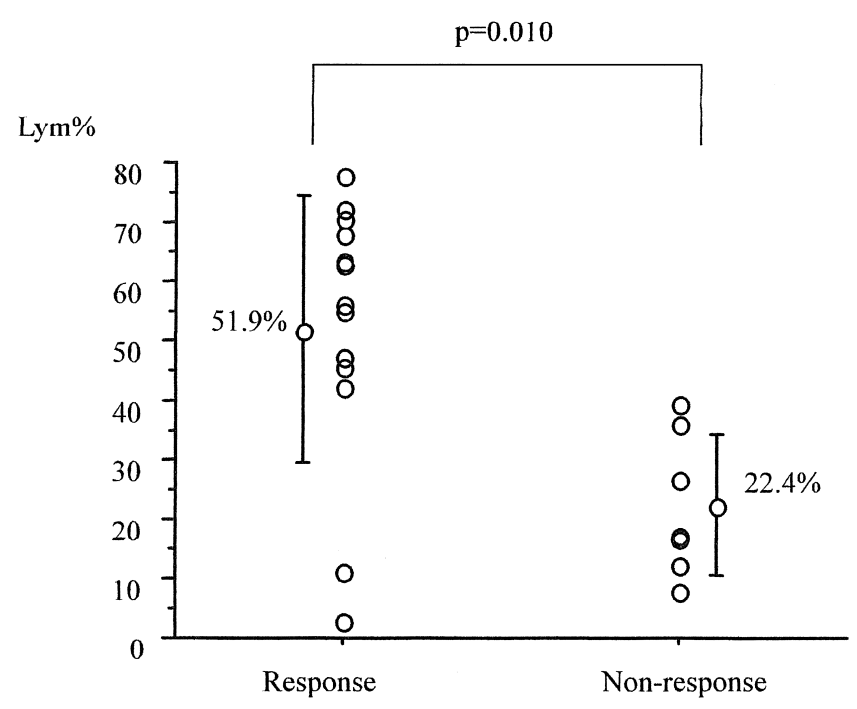

Figure 1-1. Lymphocyte rate in the response group and non-response group. There was a statistically significant difference between lymphocyte rate in the response group and in the non-response group (Mann-Whitney $U$ analysis, $\mathbf{p}=\mathbf{0 . 0 1 0})$.

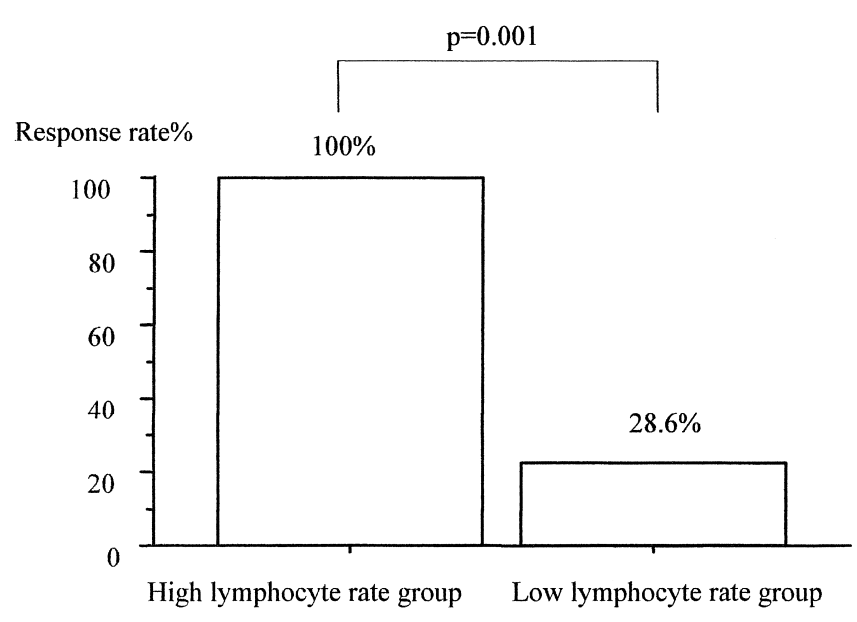

Figure 1-2. Response rate in the high lymphocyte rate group and low lymphocyte rate group. There was a statistically significant difference between response rate in the high lymphocyte rate group and in the low lymphocyte rate group (Fisher's direct probability analysis, $\mathbf{p}=\mathbf{0 . 0 0 1}$ ).

Here, we report the correlation between the lymphocyte rate in malignant pleural effusion and the therapeutic efficacy of OK-432.

\section{Patients and Methods}

We enrolled 20 lung cancer patients with malignant pleural effusion, admitted to our hospital between January 2000 and December 2004, who met the following criteria: (i) they had previously been untreated, (ii) OK-432 was administrated to the thoracic cavity, (iii) prognosis was known. Ta- 
Lym $\%$

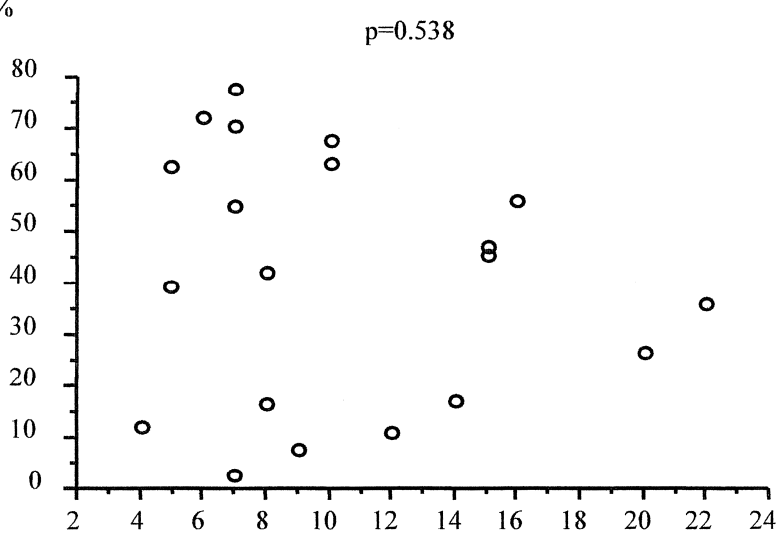

Duration of drainage (days)

Figure 2. Correlation between lymphocyte rate and duration of drainage. Lymphocyte rate was not statistically correlated with duration of drainage.

ble 1 shows the patient characteristics. The histology of the patients was mostly adenocarcinoma. The number of patients who received systemic chemotherapy was 11 . The others did not receive systemic chemotherapy since the performance status of the Eastern Cooperative Oncology Group was 3 or 4 , or they had complications. We referred to the medical records of these patients and, in a case-control study, investigated whether the lymphocyte rate influenced the therapeutic efficacy of intrapleural OK-432.

The lymphocyte rate was determined by a cytological examination of malignant pleural effusion before treatment. The rate was obtained using the number of lymphocytes and the number of nucleated cells in malignant pleural effusion.

The technique of intrapleural OK-432 administration is as follows. First, a $20 \mathrm{Fr}$ or $24 \mathrm{Fr}$ chest drainage tube is inserted into the thoracic cavity, and continuous chest drainage is performed. Next, when the discharge of pleural effusion falls below $300 \mathrm{ml} /$ day, and after it has been confirmed that pulmonary re-expansion is good, OK-432 is injected into the thoracic cavity at $10 \mathrm{KE} /$ body and the catheter is clamped. Four hours later, the clamp is opened and continuous chest drainage is again performed. If the discharge of pleural effusion falls below $100 \mathrm{ml} /$ day, the tube is removed.

The therapeutic efficacy of intrapleural OK-432 against malignant pleural effusion was assessed from the response rate, duration of chest drainage after intrapleural OK-432 (duration of drainage), time to progression of malignant pleural effusion (TTP of malignant pleural effusion), and survival time.

To evaluate the response rate, patients where disappearance or a clear decrease of pleural effusion continued for more than 4 weeks were placed in the Response group; others were placed in the Non-response group. For statistical analysis, Mann-Whitney U analysis was used to compare the lymphocyte rate. Fisher's direct probability analysis was

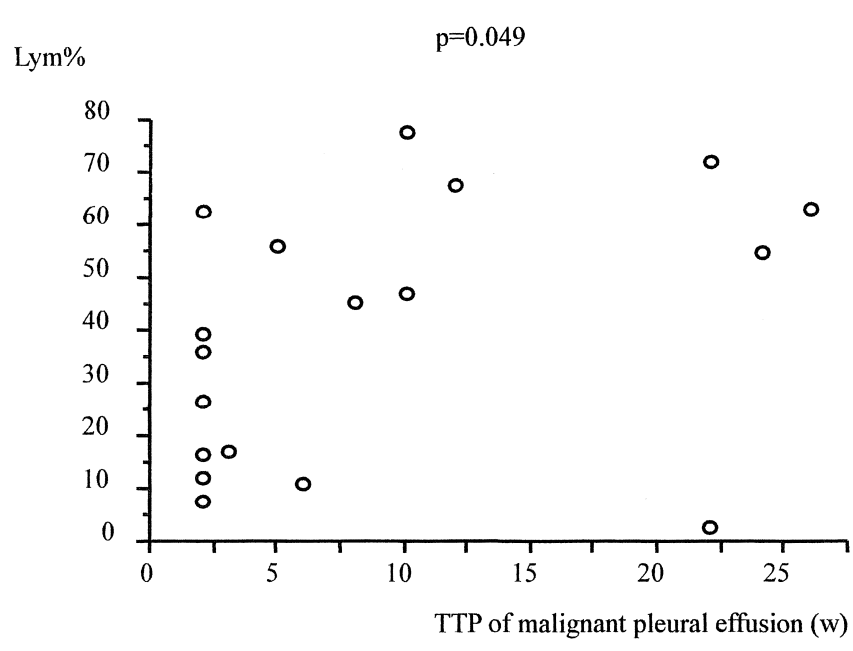

Figure 3-1. Correlation between lymphocyte rate and TTP of malignant pleural effusion. Lymphocyte rate was statistically correlated with TTP of malignant pleural effusion.

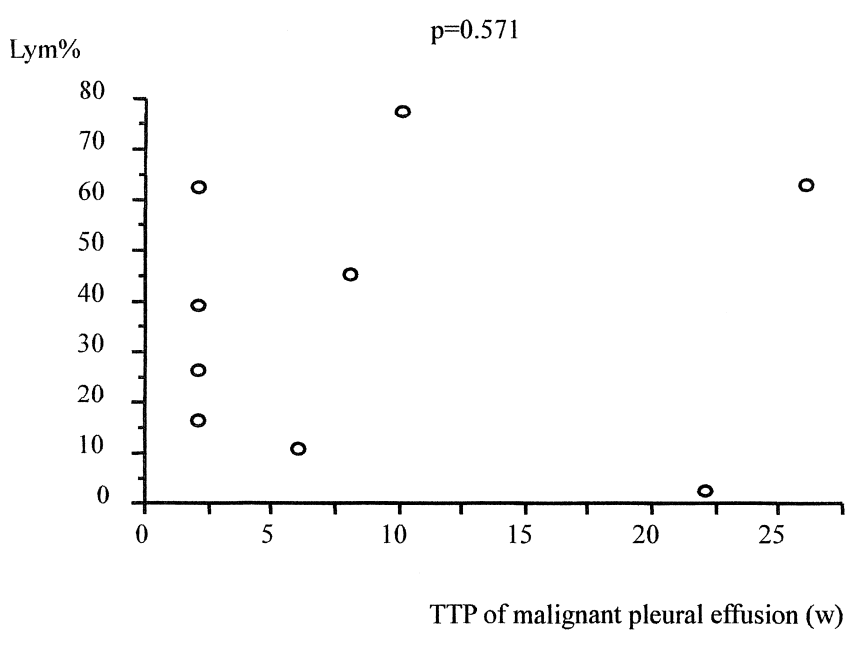

Figure 3-2. Correlation between lymphocyte rate and TTP of malignant pleural effusion in patients who had received systemic chemotherapy. Lymphocyte rate was not statistically correlated with TTP of malignant pleural effusion in patients who received systemic chemotherapy.

used to compare the response rate. To examine the correlation between the lymphocyte rate and duration of drainage, TTP of malignant pleural effusion, and survival time, a twovariable graph was made, and Spearman's rank correlation was used. $\mathrm{p}<0.05$ was designated as statistically significant. Statistical processing was done using StatView 5.0. 


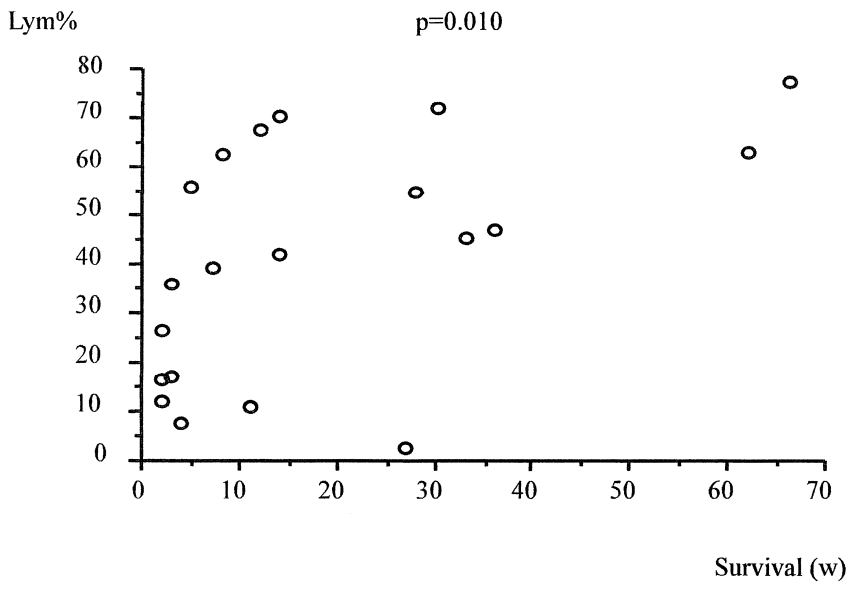

Figure 4-1. Correlation between lymphocyte rate and survival time. Lymphocyte rate was statistically correlated with survival time.

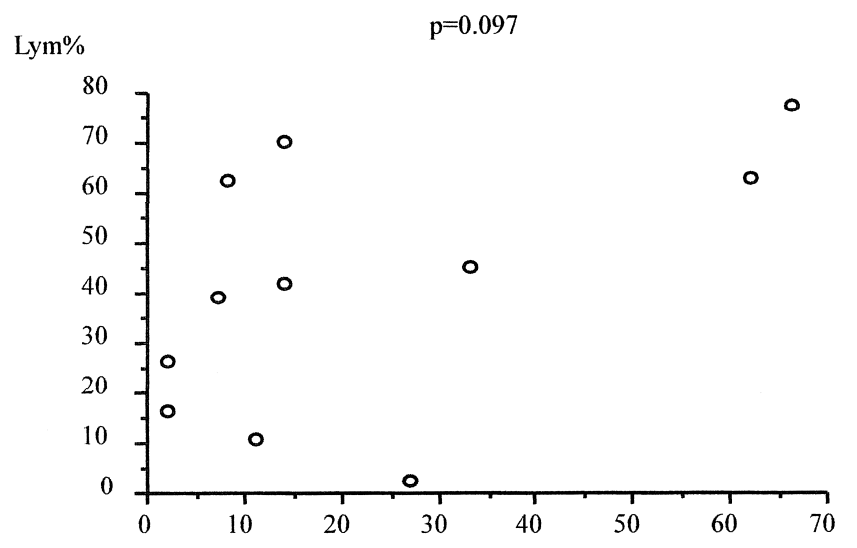

Survival (w)

Figure 4-2. Correlation between lymphocyte rate and survival time in patients who had received systemic chemotherapy. Lymphocyte rate was not statistically correlated with survival time in patients who received systemic chemotherapy.

\section{Results}

\section{Correlation between the lymphocyte rate and response rate in malignant pleural effusion}

Thirteen of 20 patients (65\%) showed a response (Response group). The others showed no response (Nonresponse group). Distributions of average age, sex, PS, and histology for the two groups were almost the same. The lymphocyte rate in the Response group's malignant pleural effusion averaged of $51.9 \%$ (3.0-78.0\%). However, the lymphocyte rate in the Non-response group averaged $22.4 \%$ (8.0-39.4\%). There was a statistically significant difference between the lymphocyte rates of these two groups (Mann-
Whitney U analysis, $\mathrm{p}=0.010$ ). The lymphocyte rate in the Response group's malignant pleural effusion was significantly higher than in the Non-response group (Fig. 1-1).

The average of lymphocyte rate in malignant pleural effusion was $41.6 \%$ in all patients. Eleven of 20 patients $(55 \%)$ had an above-average lymphocyte rate (the High lymphocyte rate group). The others had a below-average lymphocyte rate (the Low lymphocyte rate group). The response rate was $100 \%$ in the High lymphocyte rate group, whereas was only $28.6 \%$ in the Low lymphocyte rate group. Distributions of patient characteristics for the two groups were almost the same. There was a statistically significant difference between the response rates of these two groups (Fisher's direct probability analysis, $\mathrm{p}=0.001$ ). The response rate in the High lymphocyte rate group was significantly higher than in the Low lymphocyte rate group (Fig. 1-2).

\section{Correlation between the lymphocyte rate and duration of drainage in malignant pleural effu- sion}

Average of duration of drainage was 10.4 days (4-22 days). A two-variable graph was made to examine the correlation between the lymphocyte rate and duration of drainage (Fig. 2). The lymphocyte rate was not statistically correlated with the duration of drainage.

\section{Correlation between the lymphocyte rate in malignant pleural effusion and TTP of malignant pleural effusion}

The median TTP of malignant pleural effusion was nine weeks. A two-variable graph was made to examine the correlation between the lymphocyte rate and TTP of malignant pleural effusion (Fig. 3-1). The lymphocyte rate was statistically correlated with TTP of malignant pleural effusion. If the cases were restricted to those who received systemic chemotherapy, the median TTP of malignant pleural effusion was nine weeks, and the lymphocyte rate was not statistically correlated with TTP of malignant pleural effusion (Fig. 3-2).

\section{Correlation between the lymphocyte rate in malignant pleural effusion and survival time}

Median survival time was 14 weeks. A two-variable graph was made to examine the correlation between the lymphocyte rate and survival time (Fig. 4-1). The lymphocyte rate was statistically correlated with survival time. If the cases were restricted to those who received systemic chemotherapy, the median survival time was 14 weeks. The lymphocyte rate was not statistically correlated with survival time (Fig. 4-2).

\section{Discussion}

This study showed a correlation between the lymphocyte rate in malignant pleural effusion and the therapeutic efficacy of intrapleural OK-432. Lymphocytes in malignant 
pleural effusion appear in response to the presence of cancer cells. They seem to be related to antitumor immunity in the same way as tumor-infiltrating lymphocytes (TILs). TILs are various types of lymphocytes with antitumor activity. They have killer activity against autologous cancer cells. Their antitumor activity is stronger than lymphokine-activated killer (LAK) cells, which are peripheral lymphocytes activated by IL-2 (13-15). According to an analysis of cell surface antigens, TILs mainly comprise $\mathrm{CD}^{+}$and $\mathrm{CD}^{+} \mathrm{T}$ cells. The ratio of CD4 to CD8 in TILs is higher than in peripheral lymphocytes (16).

OK-432 was developed to exploit the antitumor efficacy of Streptococcus hemolyticus. It is produced by lyophilization of cultures of the low-virulence $\mathrm{Su}$ strain of group A Streptococcus pyogenes. It effectively decreases malignant pleural effusion in lung cancer patients and prolongs survival time when combined with chemotherapy in lung cancer and gastric cancer patients (17). In Japan, it is often administrated to the thoracic cavity to decrease pleural effusion as a local therapy for malignant pleural effusion. OK432 triggers the antitumor function of lymphocytes.

The lymphocyte rate in the Response group was significantly higher than in the Non-response group. The response rate in the High lymphocyte rate group was significantly higher than in the Low lymphocyte rate group. These response rates indicate antitumor efficacy. These results suggest that the antitumor efficacy of intrapleural treatment with OK-432 for malignant pleural effusion was high when the lymphocyte rate in the pleural effusion was high before treatment, whereas the antitumor efficacy was low when the lymphocyte rate was low. The lymphocyte rate in malignant pleural effusion thus influences the response rate to treatment by intrapleural OK-432.

The duration of drainage appears to show the speed of decreased pleural effusion after treatment with intrapleural OK-432. If pleural effusion decreases rapidly, the duration is short. Here, it was not statistically correlated with the lymphocyte rate. We believe that the lymphocyte rate does not influence the speed of the decrease of malignant pleural effusion as a result of OK-432.

TTP of malignant pleural effusion and the survival time were correlated with the lymphocyte rate. When the lymphocyte rate was high, they were prolonged. When the test was restricted to patients who had received systemic chemotherapy, however, TTP of malignant pleural effusion and survival time were not correlated with the lymphocyte rate, since the chemotherapy had a major influence. We think the reason for the discrepancy is as follows: In the High lymphocyte rate group, the control of pleural effusion was good because the response rate to intrapleural OK-432 was high. As a result, there were more patients who were able to re- ceive systemic chemotherapy post-intrapleural OK-432 than in the Low lymphocyte rate group. Survival time shows prognosis. The lymphocyte rate did not have any influence on the prognosis. A correlation between the level of infiltration in TILs and prognosis has been reported in melanoma $(18,19)$, gastric cancer $(20,21)$, colon cancer $(22,23)$, breast cancer (24), hepatocellular carcinoma (25), and bladder cancer (26). Ropponen et al showed that the level of infiltration in TILs is correlated with the prognosis, and when the level of infiltration is high, the prognosis is good (27). However, Mori et al showed that the level of infiltration in TILs is not correlated with the prognosis (28). Here, we also showed that the lymphocyte rate in malignant pleural effusion does not correlate with the prognosis. We suspect the reason for lymphocytes having no influence on prognosis is that lymphocytes that promote antitumor immunity and lymphocytes that inhibit antitumor immunity are mixed together in malignant pleural effusion.

In malignant pleural effusion, macrophages and cancer cells exist with lymphocytes. We studied macrophages and cancer cells as well as lymphocytes. The rate of macrophages and cancer cells in malignant pleural effusion did not have an influence on the therapeutic efficacy of OK-432 (data not shown). Although OK-432 activates macrophages in addition to lymphocytes (4), these results suggest that the rate of macrophages and cancer cells are not related to the therapeutic efficacy of OK-432.

Intrapleural administration of a chemical agent for malignant pleural effusion is a local therapy for the purpose of controlling pleural effusion. Anticancer drugs such as cisplatin (12) and sclerosing agents such as doxycycline (29) and talc (30) are among the most frequently used chemical agents in addition to OK-432. There have been very few reports on the efficacy of chemical agents for malignant pleural effusion other than those used in systemic chemotherapy. No consensus about a standard therapy for malignant pleural effusion has yet been achieved. In conclusion, this study showed that lymphocyte rate in malignant pleural effusion had an influence on the response rate to treatment by intrapleural OK-432, and that the response rate in the High lymphocyte rate group was significantly higher than in the Low lymphocyte rate group. In the High lymphocyte rate group, intrapleural OK-432 was effective against malignant pleural effusion. Our results suggest that intrapleural OK-432 is useful in patients who show a high lymphocyte rate in malignant pleural effusion before treatment. In the Low lymphocyte rate group, however, intrapleural OK-432 for malignant pleural effusion was not effective. We conclude that further studies are necessary to identify effective chemical agents other than OK-432 that would be effective in the Low lymphocyte rate group.

\section{References}

1. Burger PC, Green SB. Patient age, histological features and length of survival in patients with glioblastoma multiforme. Cancer 59:
1617-1625, 1987.

2. DeVita VT Jr, Hellman S, Rosenberg SA. Cancer: Principles and 
practice of oncology. 5th ed. Lippincott Williams \& Wilkins, Philadelphia, 1997.

3. Kishi K, Homma S, Sakamoto S, et al. Efficacious pleurodesis with OK-432 and doxorubicin against malignant pleural effusions. Eur Respir J 24: 263-266, 2004.

4. Katano M, Torisu M. New approach to management of malignant ascites with a streptococcal preparation, OK-432. II. Intraperitoneal inflammatory cell-mediated tumor cell destruction. Surgery 93: $365-373,1983$

5. Watabe S, Sendo F, Kimura S, Arai S. Activation of cytotoxic polymorphonuclear leukocytes by in vivo administration of a streptococcal preparation, OK-432. J Natl Cancer Inst 72: 13651370, 1984

6. Uchida A, Micksche M. Intrapleural administration of OK-432 in cancer patients: activation of NK cells and reduction of suppressor cells. Int J Cancer 31: 1-5, 1983

7. Hojo H, Hashimoto Y. Cytotoxic cells induced in tumor-bearing rats by a streptococcus preparation (OK-432). Gann 72: 692-699, 1981.

8. Ichimura O, Suzuki S, Saito M, Sugawara Y, Ishida N. Augmentation of interleukin 1 and interleukin 2 production by OK-432. Int J Immunopharmacol 7: 263-270, 1985.

9. Tsuchiya I, Kasahara T, Yamashita K, et al. Induction of inflammatory cytokines in the pleural effusion of cancer patients after the administration of an immunomodulator, OK-432: role of IL-8 for neutrophil infiltration. Cytokine 5: 595-603, 1993.

10. Fujimoto T, Duda RB, Szilvasi A, Chen X, Mai M, O'Donnell MA. Streptococcal preparation OK-432 is a potent inducer of IL12 and a $\mathrm{T}$ helper cell 1 dominant state. J Immunol 158: 56195626, 1997.

11. Saito M, Yamaguchi T, Ebina $T$, et al. In vivo production of immune interferon (IFN gamma) by murine spleen cells when different sensitizing antigens are used in vivo and in vitro. Cell Immunol 78: 379-386, 1983 .

12. Markman M, Cleary S, King ME. Cisplatin and cytarabine administrated intrapleurally as treatment of malignant pleural effusions. Med Pediatr Oncol 13: 191-193, 1985.

13. Nitta $T$, Yagita $H$, Azuma $T$, Sato $K$, Okumura K. Bispecific $\mathrm{f}(\mathrm{ab}) 2$ monomer prepared with anti-CD3 and anti-tumor monoclonal antibodies is most potent in induction of cytolysis of human $\mathrm{T}$ cells. Eur J Immunol 19: 1437-1441, 1989.

14. Itoh $\mathrm{K}$, Platsoucas $\mathrm{CD}$, Balch $\mathrm{CM}$. Autologous tumor specific cytotoxic $\mathrm{T}$ lymphocytes in the infiltrate of human metastatic melanomas. J Exp Med 168: 1419-1441, 1988.

15. Rosenberg SA, Spiess $P$, Lafrieniere R. A new approach to the adoptive immunotherapy of cancer with tumor-infiltrating lymphocytes. Science 233: 1318-1321, 1986.
16. Kuo SH, Chang DB, Lee YC, Lee YT, Luh KT. Tumor-infiltrating lymphocytes in non-small cell lung cancer are activated $\mathrm{T}$ lymphocytes. Respirology 3: 55-59, 1998.

17. Watanabe Y, Iwa T. Clinical value of immunotherapy with the streptococcal preparation OK-432 in non-small cell lung cancer. J Biol Response Mod 6: 169-180, 1987.

18. Straten P, Becker JC, Guldberg P, Zeuthen J. In situ T cells in melanoma. Cancer Immunol Immunother 48: 386-395, 1999.

19. Elder D. Tumor progression, early diagnosis and prognosis of melanoma. Acta Oncol 38: 535-547, 1999.

20. Black MM, Freeman C, Mork T, Harrei S, Cutler SJ. Prognostic significance of microscopic structure of gastric carcinoma and their regional lymph node. Cancer 27: 703-711, 1971.

21. Fukuda K, Tsujitani S, Maeta Y, Yamaguchi K, Ikeguchi M, Kaibara N. The expression of RCAS1 and tumor infiltrating lymphocytes in patients with T3 gastric carcinoma. Gastric Cancer 5: 220-227, 2002

22. Naito $Y$, Saito $K$, Shiiba $K$, et al. $\mathrm{CD}^{+} \mathrm{T}$ cells infiltrated within cancer cell nests as a prognostic factor in human colorectal cancer. Cancer Res 58: 3491-3494, 1998.

23. Okada K, Komuta K, Hashimoto $S$, Matsuzaki S, Kanematsu T, Koji T. Frequency of apoptosis of tumor-infiltrating lymphocytes induced by fas counterattack in human colorectal carcinoma and its correlation with prognosis. Clin Cancer Res 6: 3560-3564, 2000.

24. Nathanson L. Immunology and immunotherapy of human breast cancer. Cancer Immunol Immunother 2: 209-224, 1977.

25. Kawata A, Une Y, Hosokawa M, Uchino J, Kobayashi H. Tumorinfiltrating lymphocytes and prognosis of hepatocellular carcinoma. Jpn J Clin Oncol 22: 256-263, 1992.

26. Lipponen PK, Eskelinen MJ, Jauhiainen K, Harju E, Terho R. Tumor infiltrating lymphocytes as an independent prognostic factor in transitional cell bladder cancer. Eur J Cancer 29: 69-75, 1992.

27. Ropponen KM, Eskelinen MJ, Lipponen PK, Alhava E, Kosma VM. Prognostic value of tumorinfiltrating lymphocytes (TILs) in colorectal cancer. J Pathol 182: 318-324, 1997.

28. Mori M, Ohtani H, Naito Y, Sagawa M, Sato M, Fujimura S, Nagura $\mathrm{H}$. Infiltration of $\mathrm{CD}^{+} \mathrm{T}$ cells in non-small cell lung cancer is associated with dedifferentiation of cancer cells, but not with prognosis. Tohoku J Exp Med 191: 113-118, 2000.

29. Robinson LA, Fleming WH, Galbraith TA. Intrapleural doxycycline control of malignant pleural effusions. Ann Thorac Surg $\mathbf{5 5}$ (5): 1115-1121, 1993.

30. Fentiman IS, Rubens RD, Hayward JL. A comparison of intracavitary talc and tetracycline for the control of pleural effusions secondary to breast cancer. Eur J Cancer Oncol 22: 1079-1081, 1986.

(C) 2006 The Japanese Society of Internal Medicine http://www.naika.or.jp/imindex.html 